Check for updates

Cite this: Mater. Horiz., 2017, 4,608

Received 16th February 2017, Accepted 27th March 2017

DOI: $10.1039 / c 7 m h 00091 j$

rsc.li/materials-horizons

\section{A unified understanding of charge transport in organic semiconductors: the importance of attenuated delocalization for the carriers $\dagger$}

\author{
Chuan Liu, ${ }^{\star a}$ Kairong Huang, ${ }^{a}$ Won-Tae Park, ${ }^{b}$ Minmin Li, ${ }^{a}$ Tengzhou Yang, ${ }^{a}$ \\ Xuying Liu, ${ }^{c}$ Lijuan Liang, ${ }^{\text {cd }}$ Takeo Minari ${ }^{c}$ and Yong-Young Noh (D)*b
}

The variety of charge transport theories for organic semiconductors (OSCs) raises the question of which models should be selected for each case, and there is a lack of generalized understanding regarding various OSCs over the full range of crystallinity from single crystal to amorphous. Here, we report that the generalized Einstein relation (GER) can unify various theoretical models and predict charge transport in OSCs with various crystallinities, by altering the variance of the density of states and the delocalization degree in a Gaussian-distributed density of states. The GER also provides a good fitting to much of the experimental data of temperature- and gate-voltage-dependent mobility for different OSCs in transistors. Consequently, disorders of charge transport in various OSCs can be directly compared in the same map, which reveals how energetic disorder and the delocalization degree determine charge transport in organic devices.

\section{Introduction}

Recently, many new organic semiconductors (OSCs) have been reported to exhibit high field-effect mobility $\left(>10 \mathrm{~cm}^{2} \mathrm{~V}^{-1} \mathrm{~s}^{-1}\right),{ }^{1}$ despite the morphology of some thin films not being highly crystalline. $^{2}$ For example, it is difficult to predict the charge transport properties through checking the crystallinity of state-of-the-art donor-acceptor (D-A) conjugated polymers by X-ray-based structural analysis tools as many of them exhibit a low crystallization degree or possess a non-crystalline nature. ${ }^{3}$ New types of methods need to be developed to further understand the charge transport of these new OSCs, and a general

\footnotetext{
${ }^{a}$ State Key Laboratory of Optoelectronic Materials and Technologies and the Guangdong Province Key Laboratory of Display Material and Technology, School of Electronics and Information Technology, Sun Yat-sen University, Guangzhou 510275, China. E-mail: liuchuan5@mail.sysu.edu.cn

${ }^{b}$ Department of Energy and Materials Engineering, Dongguk University, 26 Pil-dong, 3 ga, Jung-gu, Seoul 100-715, Republic of Korea. E-mail: yynoh@dongguk.edu ${ }^{c}$ International Centre for Materials Nanoarchitectonics (WPI-MANA), National Institute for Materials Science (NIMS), Tsukuba, Ibaraki 305-0044, Japan

${ }^{d}$ Beijing Institute of Graphic Communication, Beijing 102600, China

$\dagger$ Electronic supplementary information (ESI) available. See DOI: 10.1039/c7mh00091j
}

\section{Conceptual insights}

Different classes of organic semiconductors (OSCs) have been described by various charge transport mechanisms from a model of band transport to variable range hopping, according to the molecular structure and crystallization. There is a lack of consensus in describing, predicting, and comparing transport in OSCs. This work describes a new understanding in charge transport, the generalized Einstein relation (GER), which unifies several classic theories for transport dynamics. By defining the variance in the density of states and delocalization degree, the GER predicts well the experimental data of a broad range of OSCs with various crystallinities, including temperature- and gate-voltage-dependent mobility data in transistors. The results are sufficiently general to allow a direct comparison of disorder factors in charge transport and provide guidelines to understand how energetic disorder and localization determine the electric characteristics of organic devices. consensus is still far from being reached, especially for those OSCs with high structural disorder but with superb mobility. ${ }^{4}$ Unlike single-crystals, the abundant structural defects and strong lattice vibrations owing to the weak van der Waals interactions are inherent in OSCs and result in complicated transport mechanisms, ${ }^{5}$ which lead to the dependence of the mobility on the temperature and/or the carrier concentration. ${ }^{6}$

Up to now, different theories concerning microscopic charge transport mechanisms have been proposed and many of them have been supported by evidence in different experiments on the temperature dependence of carrier mobility with various materials. ${ }^{4 a, 5 a, 6 b} \mathrm{~A}$ brief summary of charge transport theories is given in Fig. 1, in which the theories are roughly classified according to the crystallinity or the structural disorder of the semiconductors: (1) classic band-like transport is expected for disorder-free OSCs, which are mainly single crystalline materials ${ }^{7}$ or some conjugated polymers with extremely low torsions of chains. ${ }^{8}$ (2) For polycrystalline OSCs with a low extent of structural disorder, the mobility edge model is applicable and it is similar to the multiple trap and release (MTR) model. ${ }^{6 b, 9}$ (3) In amorphous or highly disordered semiconductors, charge transport mainly occurs by hopping or tunneling among localized 


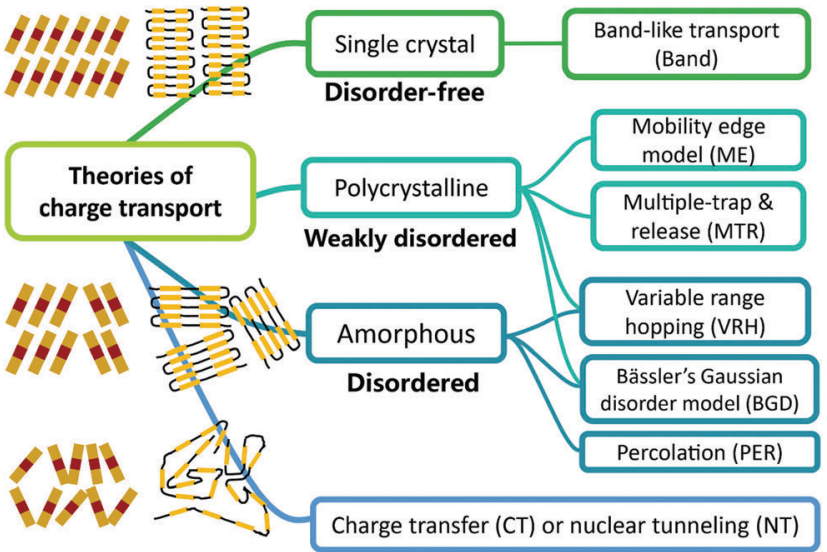

Fig. 1 A brief summary of charge transport theories in organic semiconductors. According to the degree of order or crystal structure of the semiconductor, these theories can be roughly divided into several categories.

states, and the most commonly used models are the variable range hopping model, ${ }^{10}$ Bässler's Gaussian disorder model, ${ }^{6 b, 11}$ and the percolation model. ${ }^{6 b, 9 a, 12}$ Some of them are also considered for OSCs in a polycrystalline state. (4) As in many OSCs carrier transport along the polymeric backbone would require a reorganization energy and also the polarization effect exists, thus the Marcus charge transfer (CT) model $^{13}$ should be considered. Furthermore, as the CT is driven by the coupling to nuclear vibrations (i.e. a heat bath), ${ }^{14}$ the effect of the local nuclear configurations should be taken into account, especially for non-polar OSCs where the relevant stretching vibrations are at a high frequency. This mechanism is described by the quantum nuclear tunneling (NT) theory, ${ }^{14,15}$ in which the carriers tunnel through the potential formed by the coupling of the electronic charge to its nuclear environment. Compared to the CT theory, the NT theory predicts higher values of mobility in the OSCs that are consistent with some experimental results. ${ }^{16}$

Because of the large variance of the adapted models and their limitations in specific materials, the disorders of charge transport in different microscopic mechanisms and in different polymers cannot be directly and generally compared. In addition, as mentioned above, recent studies on the high mobility of noncrystalline OSCs imply that a more general description of charge transport disorder is needed. In this report, we present a generalized charge transport model that is valid for various OSCs with different morphologies by using the so-called generalized Einstein relation (GER). By altering the variance of the density of states $(\Delta E)$ and delocalization degree $(\Delta D)$ in a Gaussian-distributed density of states, the GER can describe well most charge carrier mobility dependencies on temperature as predicted by the aforementioned theories. This is also evidenced in experiments with various OSCs from single crystal small molecules to amorphous conjugated polymers, as well as the experimentally observed dependence of mobility on the charge carrier concentration. Consequently, the charge transport in various OSCs can be regarded as being determined by $\Delta E$ and $\Delta D$, and the disorder in the charge transport can now be directly compared in the same map formed by the coordinates $\Delta E$ and $\Delta D$.

\section{Results and discussion}

\section{Description of charge transport}

For a disorder-free, single crystalline semiconductor in the nondegenerate state (i.e., using the Boltzmann approximation), the Einstein relation between the diffusion coefficient $D$ and the mobility $\mu$ takes its classical form: $D / \mu=k T / q$, where $k$ is Boltzmann's constant, $T$ is the temperature, and $q$ is the electron charge. In disordered semiconductors in the degenerate state, the ratio of the diffusion coefficient to the mobility becomes a function of the carrier concentration in the GER: ${ }^{17}$

$$
\frac{D}{\mu}=\frac{1}{q} \frac{\frac{n\left(E_{\mathrm{F}}, T\right)}{\partial n\left(E_{\mathrm{F}}, T\right)}}{\partial E_{\mathrm{F}}}
$$

where $n$ is the total carrier concentration and $E_{\mathrm{F}}$ is the Fermi energy. In OSCs, eqn (1) should be used under the assumption that the mobility can also be a function of energy $E$, for example by assuming a certain hopping probability. ${ }^{18}$ We propose that the mobility can be calculated by using the GER, which is derived according to the special features of OSCs described below.

The first important feature of OSCs is the relatively weak bonding strength and electronic interactions between neighboring molecules, which result in the density of states (DOS) having a narrow Gaussian distribution: ${ }^{17,19}$

$$
N(E)=\frac{N_{\mathrm{t}}}{\sqrt{2 \pi} \Delta E} \exp \left[-\frac{\left(E-E_{0}\right)^{2}}{2 \Delta E^{2}}\right]
$$

Here $N_{\mathrm{t}}$ is the characteristic band DOS (usually $10^{21} \mathrm{~cm}^{-3}$ ), $\Delta E$ is the variance of the Gaussian distributed DOS (usually below $0.2 \mathrm{eV})$, and $E_{0}$ is the energy of the DOS center $\left(E_{0}\right.$ is referenced as $0 \mathrm{eV}$ ). The DOS with different $\Delta E$ is illustrated in the ESI, $\dagger$ Fig. S1. The second feature of OSCs is that in transistor operation the quasi Fermi level may not always be far from the edge of the conduction bands or levels (i.e. HOMO or LUMO levels), especially when the energy levels are strongly bent by gate-voltage tuning. Thus, degeneration has to be considered and so the Fermi-Dirac distribution is used to calculate the overall carrier density $n$ :

$$
n=\int_{-\infty}^{+\infty} N(E) f(E) \mathrm{d} E=\int_{-\infty}^{+\infty} N(E)\left[1+\exp \left(\frac{E-E_{\mathrm{F}}}{k T}\right)\right]^{-1} \mathrm{~d} E
$$

The third feature of OSCs is that defects or traps are abundant, and so electronic states in tails are more localized compared to those well above the tail states (as shown in Fig. 2b). That being said, the microscopic conductivity of electronic states, $\sigma^{\prime}$, is energy-dependent rather than being constant: ${ }^{10 d, 19 b}$

$$
\sigma^{\prime}=q^{2} N(E) D(E)
$$

where $D(E)$ is the diffusivity for each energy and will be discussed below. Thus, the overall conductivity $\sigma$ for all the carriers can be calculated by the Kubo-Greenwood integral: ${ }^{10 d, 19 b}$

$$
\sigma=\int_{-\infty}^{+\infty} \sigma^{\prime}(E)\left[-\frac{\partial f(E)}{\partial E}\right] \mathrm{d} E
$$


(a)

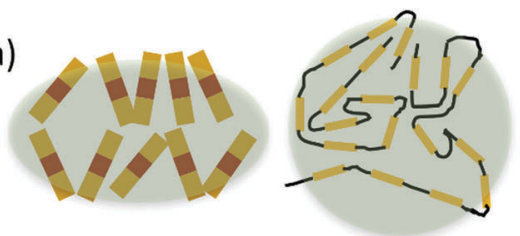

(b)

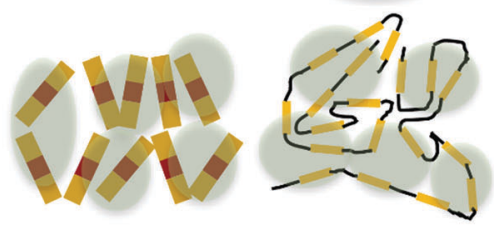

(d)

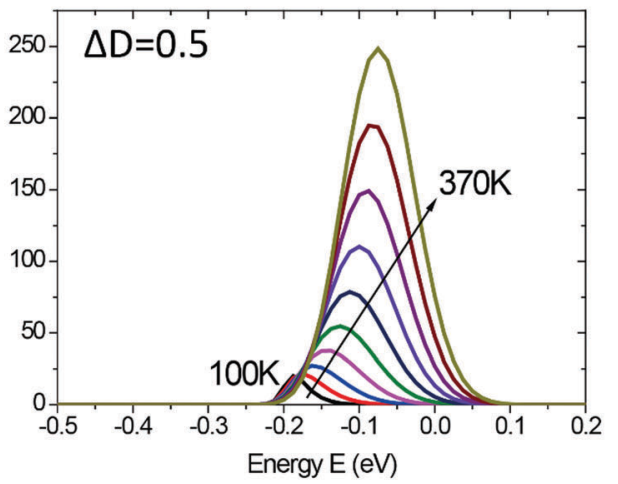

(f) $\Delta \mathrm{E}=0.20$

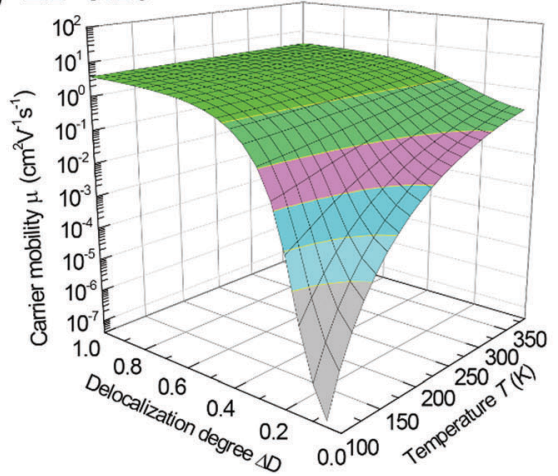

(c)

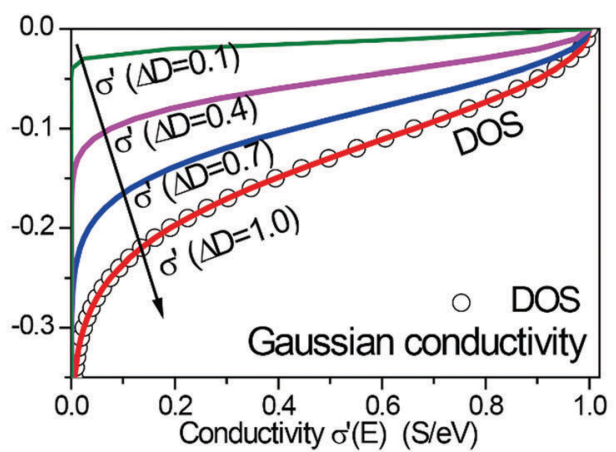

(e)

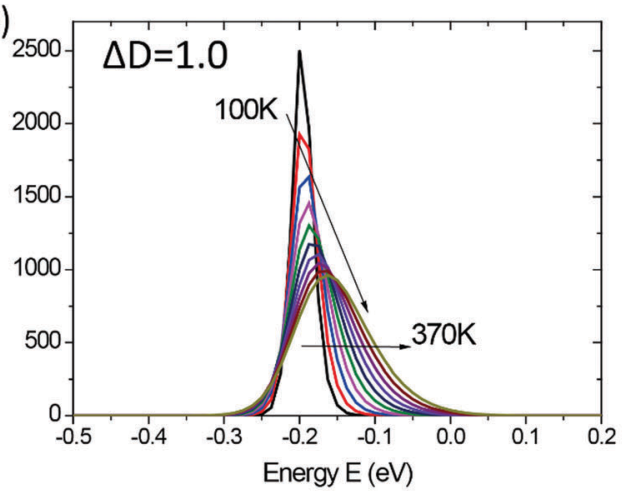

(g) $\Delta \mathrm{E}=0.06$

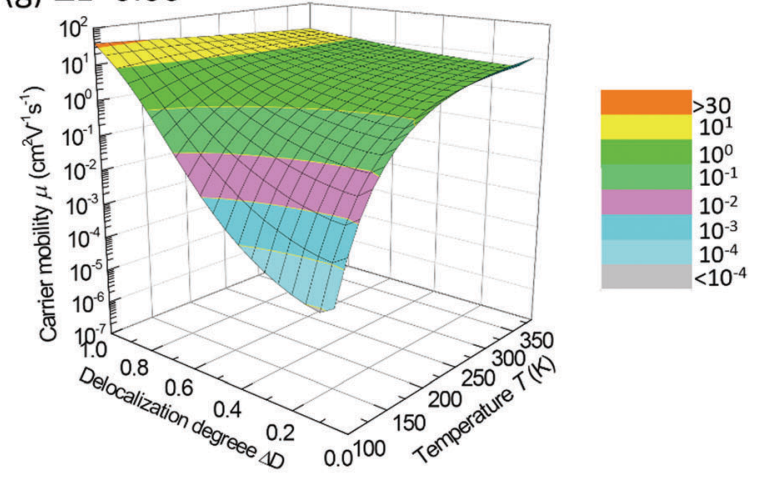

Fig. 2 (a and b) Schematic representations of the delocalization of carriers in the states with high energies near the DOS center (a) or in the states with low energies near the edge of the DOS (or near the energy gap) (b). The shades are illustrative of the delocalization area. (c) Normalized microscopic conductivity $\sigma^{\prime}(E)$ as a function of energy for different degrees of delocalization $\Delta D$ ( $\Delta E$ is fixed at $0.11 \mathrm{eV}$ ). (d) and (e) The differential conductivities $\frac{\mathrm{d} \sigma}{\mathrm{d} E}=\sigma^{\prime}(E)\left[-\frac{\partial f(E)}{\partial E}\right]$ for different temperatures with low or high delocalization degrees $(\Delta D=0.5$ or $\Delta D=1)$. The temperatures are varied from $T=100 \mathrm{~K}$ up to $370 \mathrm{~K}\left(\Delta E=0.1 \mathrm{eV}, E_{\mathrm{F}}=-0.2 \mathrm{eV}\right)$. (f) and $(\mathrm{g})$ The effective mobility as a function of temperature for various $\Delta D(\Delta E=0.20$ and $0.06 \mathrm{eV}$, respectively; $E_{\mathrm{F}}$ is fixed at $-0.2 \mathrm{eV}$ ). The effective mobility decreases as the temperature increases at $\Delta D=1$, which is consistent with band-like transport.

The above three features of OSCs in FETs, i.e. weak bonding, degeneration, and defect-abundance, are quantified by eqn (2)-(5), respectively, and we can use them to calculate the overall mobility for general OSCs by:

$$
\mu=\frac{\sigma}{q n}=\frac{q \int_{-\infty}^{+\infty} N(E) D(E)\left[-\frac{\partial f(E)}{\partial E}\right] \mathrm{d} E}{\int_{-\infty}^{+\infty} N(E) f(E) \mathrm{d} E}
$$

Eqn (6) quantifies the relationship between mobility and diffusivity, and thus provides a GER for disordered materials and especially for OSCs.
The diffusivity for each energy $D(E)$ in eqn (4) should be defined considering the microscopic mechanisms of charge transport in OSC solids. On one hand, in perfect crystals the electronic orbits and the delocalization of charges within a molecule or among any adjacent molecules are identical throughout the solids, respectively, and together lead to the intrinsic transport properties of the electronic states in the whole DOS (Fig. 2a). In this case, the macroscopic charge transport properties would ideally follow the first-principles evaluations. ${ }^{16 a, 20}$ On the other hand, as microscopic voids, grain boundaries, polymeric twisting, or chemical impurities are often the origin of a large amount of tail states in the DOS, these states near the energy 
gap may exhibit transport properties seriously deviating from the intrinsic transport characteristics of the states near the center of the DOS, and they may possess a reduced delocalization length (or diffusion length) as compared to that predicted by the first-principles evaluations in the microscopic results (Fig. 2b). To this end, the attenuation of conductivity from the DOS center to the edge should be considered and, for generality, the diffusivity is assumed to be Gaussian-like ${ }^{19 a}$ and then $\sigma^{\prime}(E)$ can be described by:

$$
\sigma^{\prime}(E)=\frac{\sigma_{0}}{\sqrt{2 \pi}(\Delta D \cdot \Delta E)} \exp \left[-\frac{\left(E-E_{0}\right)^{2}}{2(\Delta D \cdot \Delta E)^{2}}\right]
$$

where $\sigma_{0}=N_{\mathrm{C}} q \mu_{0} / 2$ is the characteristic conductivity and $\mu_{0}$ is the characteristic mobility, which are material-specific. The characteristic parameter $\Delta D(\leq 1$, no unit) reflects the degree of delocalization for the electronic states near the edge of the DOS (near HOMO or LUMO levels): a small $\Delta D$ means a severely attenuated $\sigma^{\prime}(E)$ from the center of the DOS (extended states) to the edge (localized states), and thus the tail states possess a much lower microscopic conductivity (i.e. severely localized) than those near the center states. When $\Delta D=1$, the delocalization degree is the same for the whole DOS and the shape of $\sigma^{\prime}(E)$ is only determined by the DOS (Fig. 2c). For a reference, in the mobility edge (ME) model the states are divided into the delocalized states $\left(\mu=\mu_{0}\right)$ when $E \geq$ ME and the totally localized tail states $(\mu=0)$ when $E<\mathrm{ME}$ (ME denotes the energy as the mobility edge, see Fig. S2, ESI $\dagger$ ). Thus, above the ME, the diffusivity is constant and the microscopic conductivity is only determined by the DOS.

The above discussions do not rely on specific transport mechanisms and should apply to carrier transport in typical OSCs. It is worth noting that $\Delta E$ characterizes the degree of disorder in the energy structure and $\Delta D$ characterizes the degree of delocalization. Specifically, $\Delta E$ of the DOS is determined by the static or structural factors, including the crystallinity of the structure and the electronic interactions/coupling among neighboring molecules, while $\Delta D$ can be affected by similar factors as well as dynamic or external factors, such as lattice vibrations, scattering mechanisms, and properties of defects or traps. By only varying one parameter at a time, the differential conductivity $\frac{\mathrm{d} \sigma}{\mathrm{d} E}=\sigma^{\prime}(E)\left[-\frac{\partial f(E)}{\partial E}\right]$ is plotted against energy $E$ in Fig. $2 \mathrm{~d}$ and e. When the temperatures are varied from $T=100 \mathrm{~K}$ up to $370 \mathrm{~K}$, the maximum of $\frac{\mathrm{d} \sigma}{\mathrm{d} E}$ increases homogeneously as the temperature increases in the case when $\Delta D=0.5$, and so does the overall conductivity (Fig. $2 \mathrm{~d}$ ). In comparison, in the case when $\Delta D=1$, the maximum of $\frac{\mathrm{d} \sigma}{\mathrm{d} E}$ decreases as the temperature increases and the change in the overall conductivity becomes much smaller (Fig. 2e). The relationship between the mobility $\mu$ and the temperature $T$ ( $\mu-T$ relation) is then calculated in Fig. $2 \mathrm{f}$ and $\mathrm{g}$, where the mobility at a certain temperature with varying $\Delta D$ or vice versa can be directly read. At a certain temperature, a larger DOS variance $\Delta E$ or a smaller delocalization degree $\Delta D$ results in a decreased mobility, which is highly consistent with the above physical understanding. As the temperature decreases, the mobility degrades sharply (or raises slightly) with a low (or high) delocalization degree, which is consistent with the transport mechanism being highly limited by thermal activation (or by lattice/phonon scattering). Importantly, the GER can explain the transition from the bandlike temperature dependence of the mobility (i.e., $\mathrm{d} \mu / \mathrm{d} T<0$ ) to the thermally activated temperature dependence (i.e., $\mathrm{d} \mu / \mathrm{d} T>0$ ), which has been reported in some high mobility semiconductors in previous experiments ${ }^{21}$ and will be discussed below.

\section{Various transport models}

The relationship between the GER and the various charge transport mechanisms are studied in Fig. 3. According to the predictions from these theories, the $\mu-T$ relations are calculated for different transport models (symbols). For crystalline semiconductors, the mechanisms include band-like transport for single crystals (Fig. 3a and b) and for poly-crystals the multiple trapping and release (MTR) model, which takes a similar form to the mobility edge (ME) model (Fig. 3c and d). For amorphous semiconductors, the variable range hopping (VRH) model (Fig. 3e and f), Bässler's Gaussian disorder (BGD) model (Fig. $3 \mathrm{~g}$ and $\mathrm{h}$ ), and the percolation (PER) model (Fig. S3, ESI $\dagger$ ) are mainly involved. The Marcus charge transfer (CT) model (Fig. 3i and j) and nuclear tunneling (NT) models (Fig. 3k and l) describe the reorganization effects in OSCs due to the coupling between charges and the local environment. These models have different views of microscopic dynamic processes and the factors in charge transport, and they have been applied to various semiconductors. ${ }^{6 b, 9-15}$ In each model, the key factors determining the $\mu-T$ relation are varied while keeping other parameters constant and these factors are provided along with the model (Fig. 3b, d, f, h, j and l). For instance, in the MTR model the mean energy of the trap states $E_{\mathrm{T}}$ is varied to generate a series of data, and in the NT model $\alpha_{\mathrm{K}}$ (the Kondo parameter) is varied to describe the different coupling strengths between the charges and the aforementioned heat bath (i.e. nuclear vibrations). ${ }^{15 a}$ The ranges of these parameters were chosen essentially according to the values reported in previous literature. The $\mu-1 / T$ relations described by the above mechanisms are fitted to the GER by varying $\Delta E$ and $\Delta D$ (lines). The fitting parameters are listed in Table S1 (ESI $\dagger$ ) and the $\mu-T$ relations are also presented for a reference (Fig. S4, ESI $\dagger$ ). In general, the fittings by the GER are reasonable over a large temperature range and deviations only occur at very low temperatures. Hence, the GER generally covers various transport behaviors and provides comparisons for charge transport in different mechanisms or materials.

The values of the DOS variances $\Delta E$ and the delocalization degree $\Delta D$ are plotted for various transport mechanisms together with the corresponding key parameters in Fig. $3 \mathrm{~m}$. For example, in the VRH model $T_{1}$ is defined as $\frac{128}{9 k} \frac{\pi \alpha^{3}}{N_{\mathrm{F}}}$, where $\alpha$ is the size of the localized state and $N_{\mathrm{F}}$ is the density of states at the Fermi edge. In all of the cases, $\Delta E$ increases and $\Delta D$ decreases as those limiting factors increase, which is consistent with the physical mechanisms of these models. The DOS variance $\Delta E$ can be taken as a signature of energetic disorder, as it generally follows the energy disorder parameters in the different models, for example, $T_{1}$ in the VRH model is proportional 
(a) Band-like transport

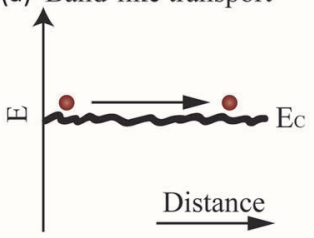

$\mu \propto T^{a}$

(e) Variable range hopping

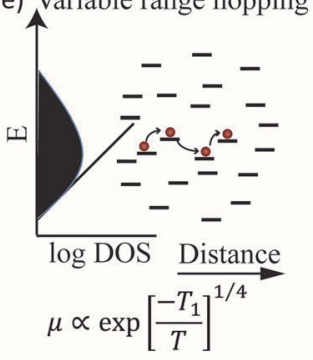

(i) Charge transfer (Marcus)

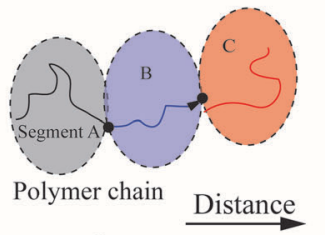

$\mu=\frac{q \alpha^{2}}{k_{B} T} k_{i f}\left(V_{i f}, \Delta G, \lambda, T\right)$
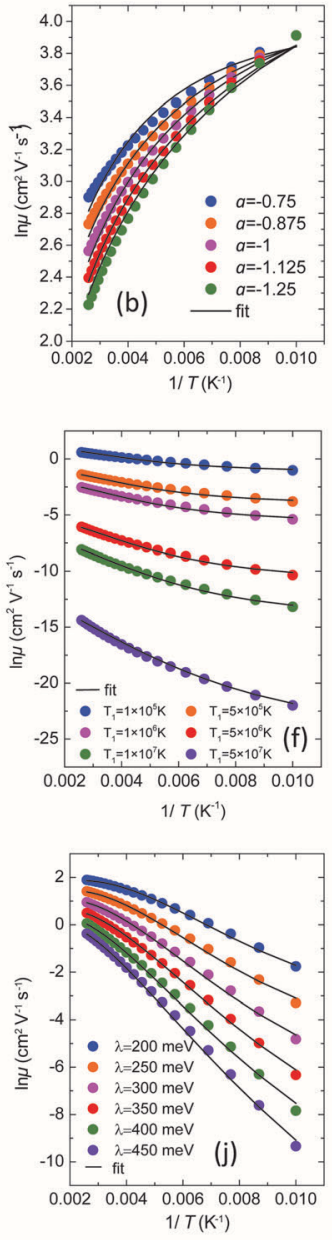

(c) Multiple-trap \& release

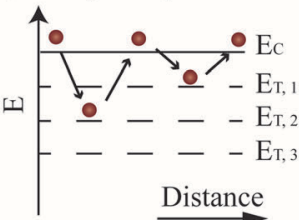

$$
\mu=\mu_{0} \alpha \exp \left(-\frac{E_{T}}{k_{B} T}\right)
$$

(g)

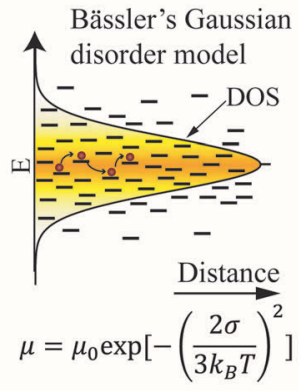

(k) Nuclear tunneling

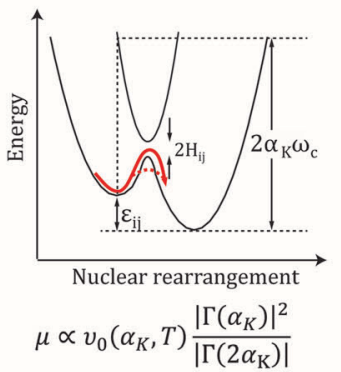

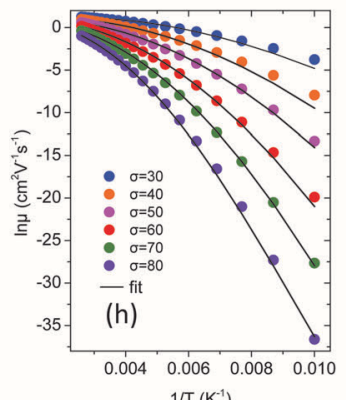
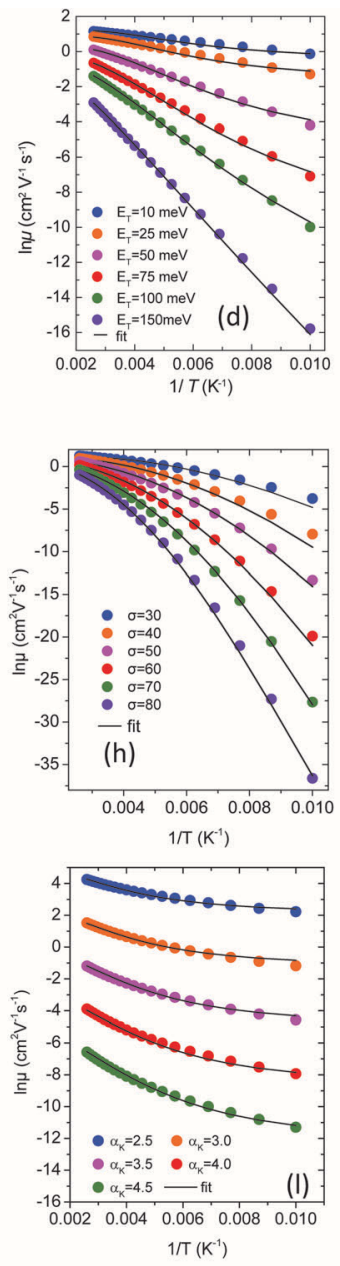

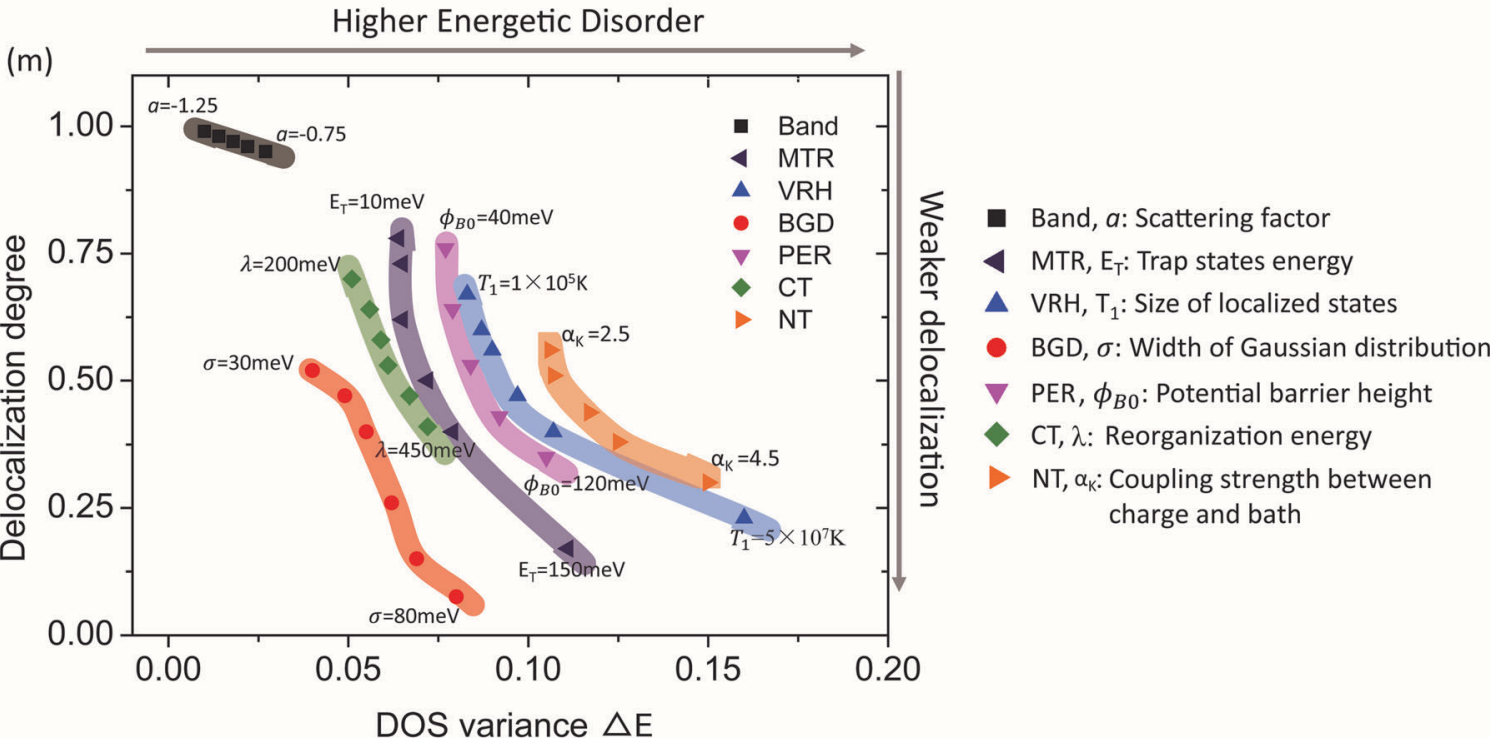

Fig. 3 Schematic representation of transport mechanisms for (a) band-like transport, (c) the multiple trap and release (MTR) model, (e) the variable range hopping (VRH) model (sketched in ref. 22), (g) Bässler's Gaussian disorder (BGD) model (sketched in ref. 11c), (i) the Marcus charge transfer (CT) model (plotted in ref. 13d), and (k) the nuclear tunneling model (NT), sketched in ref. 14 and 15 and the percolation (PER) model is given in Fig. S3 (ESI $\dagger$ ) (PER, sketched in ref. 12). The temperature dependence of the mobility calculated by different transport models is plotted with symbols and the fitting by the GER is plotted with lines: (b) band model with varying scattering factor $a$. (d) The MTR model with varying mean energy of the trap states $E_{\mathrm{T}}$. (f) The VRH model with varying $T_{1}$, proportional to the size of localized states. (h) The BGD model with varying width of the Gaussian function $\sigma$. (j) The CT model with varying reorganization energy $\lambda$. (l) The NT model with varying the Kondo parameter $\alpha_{\mathrm{K}}$. (m) The values of delocalization degree $\Delta D$ and DOS variance $\Delta E$ parameters are plotted for all of the models with the corresponding key parameters. 
to the Urbach energy. ${ }^{23}$ By gathering all of the fitting values together in Fig. 3m, the impacts of different limiting factors in the various mechanisms of charge transport can now be quantitatively compared under the same axes constructed by $\Delta E$ and $\Delta D$. Note that the CT model describes charge transport as being relatively concentrated in a low-disorder and high-delocalization area, while Bässler's Gaussian disorder (BGD) model covers the range in relatively lower degrees of delocalization. This is probably because the CT model mainly describes charge transport for polycrystalline polymers with a long conjugation length and short localized segments, while BGD is proposed for amorphous semiconductors where hopping among localized states is the main process.

\section{Experimentally measured mobility}

The GER was used to investigate the experimental data of $\mu-T$ measured for organic field-effect transistors (OFETs) made with different types of OSCs (Fig. 4). The studied materials included various OSCs from crystalline to amorphous: rubrene (Fig. 4a); ${ }^{7 a}$ pentacene (Fig. 4b) ${ }^{24}$; 6,13-bistriisopropylsilylethynyl pentacene (TIPS-PEN, ${ }^{25}$ Fig. 4c); $N$-alkyl perylene diimides (PTCDI, Fig. $4 \mathrm{~d}$ ); ${ }^{26}$ a DPP-containing copolymer with siloxane-terminated butyl or pentyl chains (PTDPPSe-SiC4 and PTDPPSe-SiC5, Fig. 4e); ${ }^{27}$ cyclopentadithiophene-benzothiadiazole (CDT-BTZ-C12, Fig. 4f); ${ }^{28}$ poly(3-hexylthiophene) (P3HT, Fig. 4g); ${ }^{29}$ poly(2,5-bis(3-alkylthiophen-2-yl)thieno(3,2-b)thiophene) (PBTTT, Fig. 4h); ${ }^{30}\left[N, N^{\prime}\right.$-bis(2octyldodecyl)-1,4,5,8-naphthalenedicarboximide-2,6-diyl]-alt-5,5'(2,2'-bithiophene) P(NDI2OD-T2) (Fig. 4i); ${ }^{31}$ and a D-A polymer containing diketopyrrolopyrrole (DPP), benzothiadiazole (BT), and selenophene (Se) (PSeDPPBT, Fig. 4j). ${ }^{32}$ The temperature dependence of the materials including copper hexadecafluorophthalocyanine $\left(\mathrm{F}_{16} \mathrm{CuPC}\right){ }^{33}$ phenyl-C61-butyric acid methyl ester (PCBM), ${ }^{34}$ dioctylbenzothienobenzothiophene (C8-BTBT) single crystals, ${ }^{1 b}$ pentacene, ${ }^{35}$ poly $\left(9,9^{\prime}\right.$-dioctylfluorene-co-bithiophene $)$ (F8T2), ${ }^{36}$ polytriarylamines (PTAA) ${ }^{30,37}$ and other OFETs is shown in Fig. S5 (ESI $\dagger$ ). For FETs with the same semiconductors, the semiconductors were fabricated in varying crystalline states or combined with various dielectric layers. The fittings from the GER calculations are shown in Table S2 (ESI $\dagger$ ), which indicates generally good fitting of the measured mobility against temperature for the different materials (lines in Fig. 4a-j).

The values used for $\Delta E$ and $\Delta D$ of the various materials are compared in Fig. 5. Small molecules are drawn in filled dots and polymers are in open dots or lines, of which the polarity of carriers is given after the materials' name. It is worth noting that the GER gives a unified description for the transition from a negative temperature coefficient of the mobility $(\mathrm{d} \mu / \mathrm{d} T<0)$ to a positive coefficient $(\mathrm{d} \mu / \mathrm{d} T>0)$, as shown in TIPS-pentacene crystals, of which $\Delta D$ is around 0.80 . It is highly consistent with and gives a quantitative explanation to the previous report which shows that the band-like temperature dependence is not due to extended-state conduction but to localized transport limited by thermal lattice fluctuations. ${ }^{21 a}$ In addition, from the mapping, it can be directly and clearly seen how the crystallization, dielectric materials, or device structures induce different charge transport disorder for the same OSC, and it can also provide comparisons in charge transport among different OSCs with different crystallinities. The small molecule rubrene crystals with different dielectrics feature distinct transport properties. This has been explained in
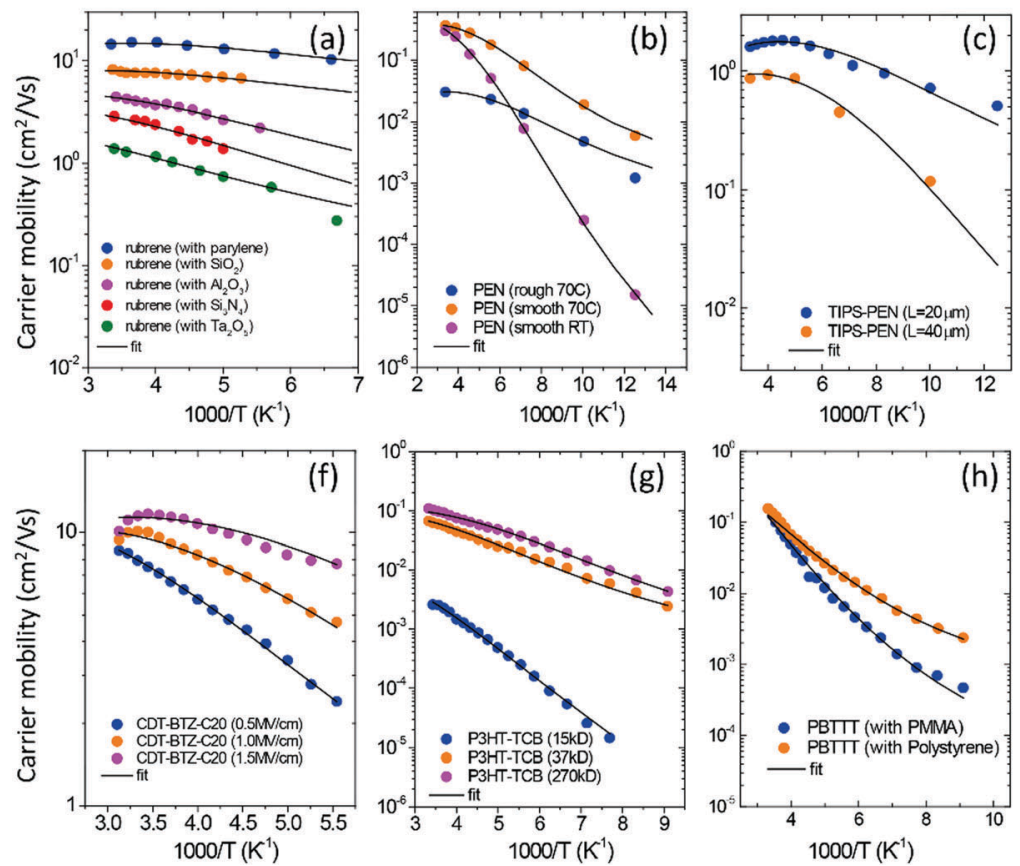
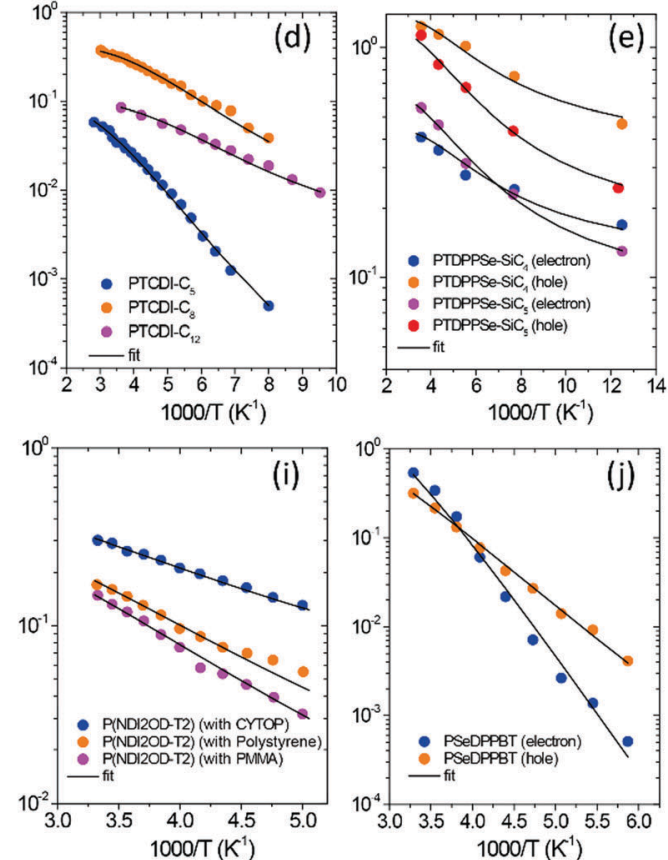

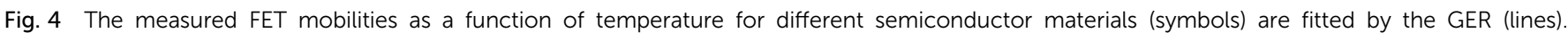

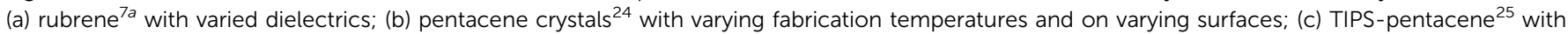

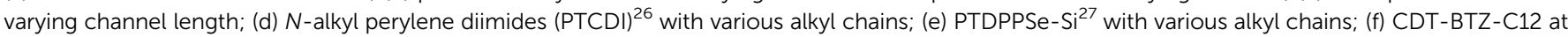

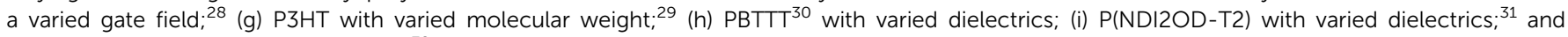
(j) PSeDPPBT with electrons and holes. ${ }^{32}$ Data for other OFETs and all of the fitting parameters are shown in ESI. $\dagger$ 

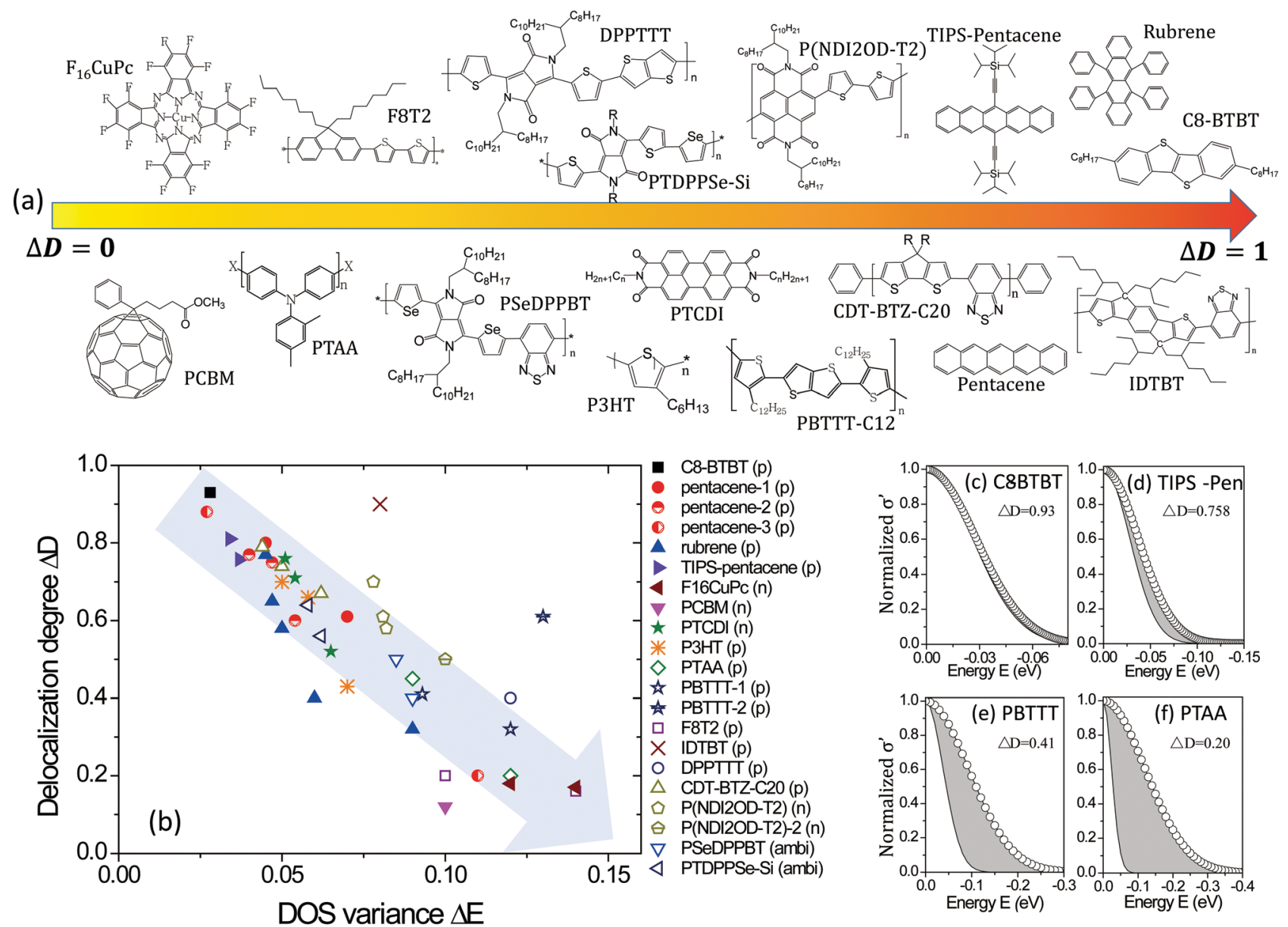

Fig. 5 (a) Schematic representations of materials roughly from a low to high delocalization degree. (b) The values of the delocalization degree $\Delta D$ and the DOS variance $\Delta E$ are plotted for different semiconductors. The grey arrow shows the direction of increasing disorder in the charge transport. (c-f) The microscopic conductivity and the DOS for some materials, where the grey areas represent the reduced microscopic conductivity owing to the localization of charge transport.

terms of the tunable small polarons ${ }^{7 a}$ and here it can be quantified that high- $k$ dielectrics noticeably decrease the delocalization degree $\Delta D$ from 0.77 (parylene) to $0.32\left(\mathrm{Ta}_{2} \mathrm{O}_{5}\right)$ and slightly broaden the DOS. Generally, for the same semiconductors with different dielectrics, e.g., TIPS-pentacene, PBTTT, and PTAA, the value of $\Delta D$ generally decreases with $\Delta E$. The reason is probably as aforementioned: the DOS variance reflecting energetic disorder is affected by factors such as structural disorders and electronic interaction/coupling between neighboring molecules or polymer chains, which also affect the hopping probability or transfer rate of charges and thus the degree of delocalization.

Interestingly, now it is possible to use the mapping to compare the transport disorder of semiconductors in different classes of materials. The investigated materials are presented in Fig. 5a roughly in the order from a low to high delocalization degree. It is worth noting that only a few D-A semiconductors exhibit high delocalization degrees, implying there is space for improvement for many D-A semiconductors by presumably optimizing the morphology or dielectric interfaces. Moreover, although thermally evaporated small molecular crystals have higher delocalization degrees and smaller energetic disorders than the solution-processed polymeric semiconductors, solution-cast films can feature very high delocalization degrees, such as C8-BTBT crystals formed by solvent vapor annealing and the polymer indacenodithiopheneco-benzothiadiazole (IDTBT) film deposited by spin-coating. The latter indicates that polymeric semiconductors would not be critically limited by crystallization but can achieve a delocalized degree close to one if they feature a rigid backbone or extended aggregates to build a transport path for intra- and inter-chain or inter-domains, ${ }^{8,38}$ respectively.

\section{Carrier-concentration-dependent mobility}

Besides temperature-dependence, the charge transport mechanism also affects the dependence of carrier mobility on the carrier numbers $(n)$ or the gate voltage $\left(V_{\mathrm{g}}\right)$ in FETs, which has also been widely observed and discussed. The dependence of mobility on $n$ or $V_{\mathrm{g}}$ is in fact attributed to the shift of $E_{\mathrm{F}}$ mainly tuned by the gate voltage. The relation between $n, V_{g}$, and $E_{\mathrm{F}}$ is given by:

$$
C_{\mathrm{i}}\left(V_{\mathrm{g}}-V_{\text {on }}\right) / q d=n=\int_{-\infty}^{+\infty} N(E) f\left(E, E_{\mathrm{F}}, T\right) \mathrm{d} E
$$


where $C_{\mathrm{i}}$ is the capacitance of the gate dielectric, $V_{\mathrm{g}}$ is the gate voltage, $V_{\text {on }}$ is the turn-on voltage, $d$ is the approximate accumulation layer thickness, and the carrier concentration owing to field-effect accumulation is $n$. We can then calculate $E_{\mathrm{F}}$ at different $V_{\mathrm{g}}$ to give the relationship between the charge mobility in the GER. The calculated mobility with varying delocalization degree $\Delta D$ is shown in the ESI $\dagger$ (Fig. S6) and the normalized mobility as a function of $V_{\mathrm{g}}$ is shown in Fig. 6a. It can be seen that the widely used power law of mobility $\mu \propto\left(V_{\mathrm{g}}-V_{\mathrm{th}}\right)^{\beta 39}$ can describe the cases at which
$\Delta D=0.2-0.8$, while the mobility becomes $V_{\mathrm{g}}$-independent or even decreases as $V_{\mathrm{g}}$ decreases at a high delocalization degree $(\Delta D=0.9$ to 1.0$)$. Furthermore, the increased power factor $\beta$ is related to a more severely attenuated delocalization near the energy gap (decreased $\Delta D$, Fig. 6b), which is in good accordance with previous reports in both theoretical and experimental studies. $^{23}$ The different $\mu-V_{\mathrm{g}}$ relationship originates from the electrostatic doping in OSCs induced by energy level bending by the gate field, which gradually shifts the quasi-Fermi level
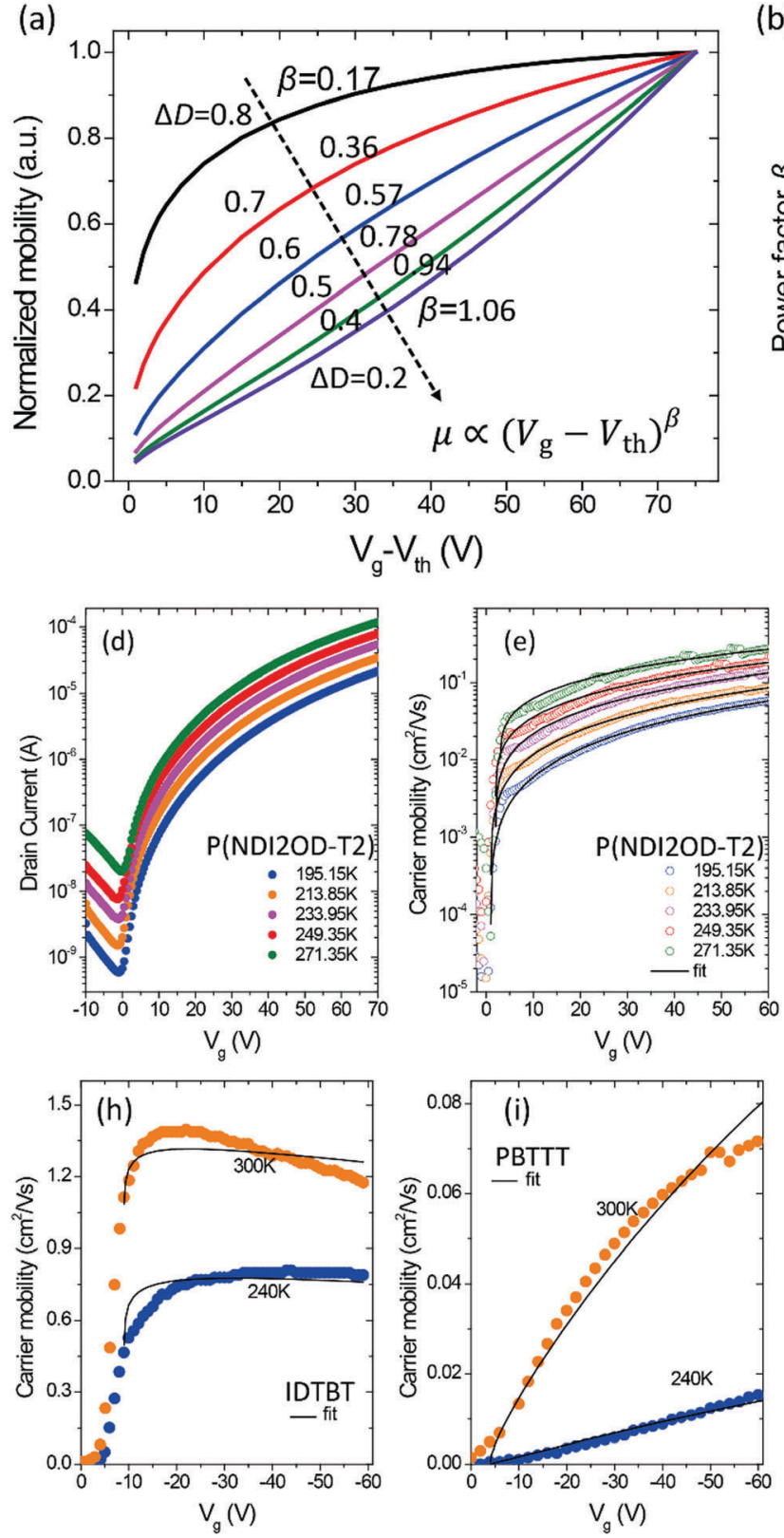
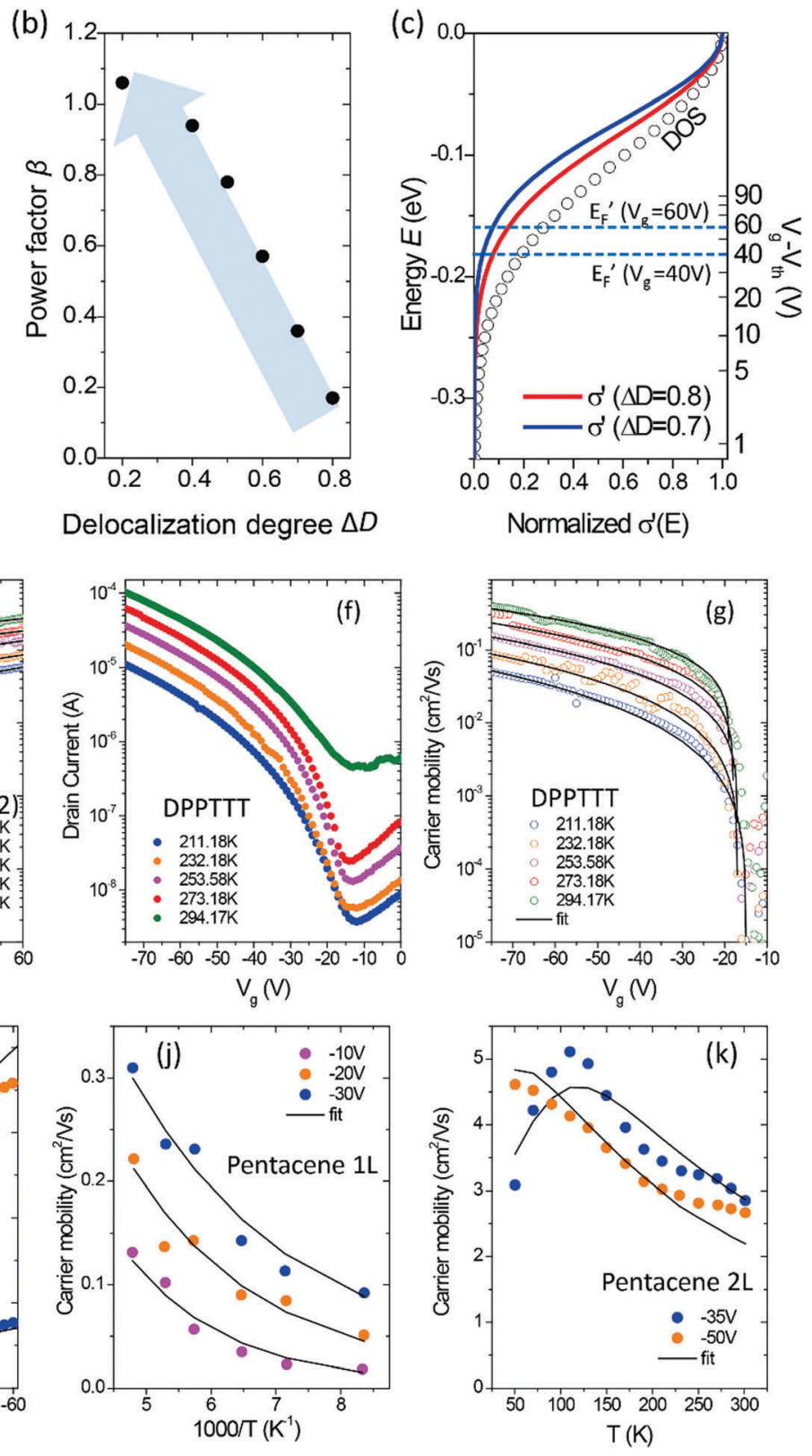

Fig. 6 (a) The calculated mobility as a function of the gate voltage in the GER for different degrees of delocalization $\Delta D$ ( $\Delta E$ is fixed at $0.1 \mathrm{eV}$ and $C_{i}=2 \times 10^{-8} \mathrm{~F} \mathrm{~cm}^{-2}$ ). The values of $\beta$ by fitting are given next to the corresponding curves. (b) The relationship between power factor $\beta$ and the delocalization degree $\Delta D$. (c) The effect of different gate voltages on the quasi-Fermi energy and the microscopic conductivity. (d-k) Data of mobility as a function of gate voltage for different semiconductor materials at various temperatures (symbols) are fitted by the GER (lines). (d and e) Drain current and extracted mobility for P(NDI2OD-T2), respectively. ( $f$ and g) Drain current and extracted mobility for DPPT-TT, respectively. The transistors were measured with $V_{D}=60 \mathrm{~V}$ and $V_{D}=-80 \mathrm{~V}$, respectively. (h) IDTBT, (i) PBTTT, (j) the first conducting layer (1L) of epitaxial-grown single crystal pentacene, and $(\mathrm{k})$ the second conducting layer (2L). Data were obtained by experimental measurements or from literature. . $^{8,40}$ 
toward transport levels at the dielectric interface, as shown in Fig. 6c. Apparently, as only the electronic states below $E_{\mathrm{F}}^{\prime}$ contribute to the channel conductance, OSCs with a smaller $\Delta D$ afford a lower overall conductivity at a small $V_{\mathrm{g}}$, and a shift of $E_{\mathrm{F}}{ }^{\prime}$ would result in a more obvious change in the overall mobility as compared with those with larger $\Delta D$.

The above GER calculation is then applied to the experimental data of the $V_{\mathrm{g}}$-dependence measured from OFETs. Transistors with the n-channel P(NDI2OD-T2) or the p-channel DPP-based copolymer DPPT-TT were fabricated as described in the experimental sections, and the transfer characteristics and the extracted mobility are shown in Fig. 6d-g. For precision, the temperature dependence as well as the $V_{\mathrm{g}}$-dependence of the mobility are simultaneously fitted by the GER. Despite the simplicity of the calculation method, the same set of parameters of $\Delta D$ and $\Delta E$ gives a reasonable fitting of the $V_{\mathrm{g}}$-dependent mobility values at varied temperatures. The values of $\Delta D$ and $\Delta E$ are listed in Table S3 (ESI $\dagger$ ). Also, the recently reported highly planar conjugated polymer IDTBT ${ }^{8}$ and epitaxial-grown single crystal (pentacene of different layer numbers) ${ }^{40}$ are investigated by the GER (Fig. 6h-k). IDTBT shows a much higher delocalization degree and a smaller DOS variance (energetic disorder) than PBTTT (see Table S3, ESI $\dagger$ ). The results agree well with the thermo-electric and spectroscopic measurements by Venkateshvaran et $a l^{8}$ In another comparison, the two-layer pentacene crystal exhibits a remarkably higher value of $\Delta D(0.88)$ than the one-layer pentacene crystal $(\Delta D=0.20)$. Hence, the GER quantitatively explains the transition from hopping to band-like transport in the different layers of pentacene mainly by the different delocalization degree, which was caused by the different quality and orientation of the crystals in the two layers according to the reports by Zhang et al. ${ }^{40}$

\section{Conclusions}

In conclusion, we have presented the GER that characterizes charge transport with a DOS variance factor $\Delta E$ and a delocalization factor $\Delta D$. By taking into account the attenuated delocalization at the electronic states near the band edge or the energy gap, we found that it can describe the carrier mobility at variable temperatures, which is predicted by various transport mechanisms. In addition, the GER is capable of describing variable carrier concentrations (various gate voltages in OFETs) of different OSCs from theoretical and experimental aspects. The GER can describe the mobilitytemperature behavior and mobility-carrier-concentration dependence predicted by various transport mechanisms in different OSCs. By using the GER to analyze the mobility, we are able to quantitatively compare charge transport properties in the degree of energetic disorder and delocalization, even for different microscopic transport mechanisms and for different classes of OSCs.

\section{Experimental section}

\section{Fabrication of transistors}

Corning Eagle 2000 glass substrates were cleaned sequentially in an ultrasonic bath with de-ionised water, acetone, and isopropanol for $10 \mathrm{~min}$ each. The $\mathrm{Au} / \mathrm{Ni}(15 \mathrm{~nm} / 3 \mathrm{~nm})$ source and drain (S/D) patterns were formed by a conventional lift-off photolithography procedure. Materials were synthesized or purchased as described in ref. 11a. The semiconductors were dissolved in 1,2-dichlorobenzene or $p$-xylene and spin-coated in nitrogen, and the thin films were then thermally annealed at $150{ }^{\circ} \mathrm{C}$ for $\mathrm{P}(\mathrm{NDI} 2 \mathrm{OD}-\mathrm{T} 2)$ or $250{ }^{\circ} \mathrm{C}$ for DPPT-TT, for $30 \mathrm{~min}$. PMMA (Aldrich, $M_{\mathrm{w}}=120 \mathrm{kD}$ ) was used as a dielectric material without further purification and was dissolved in $n$-butyl acetate $80 \mathrm{mg} \mathrm{ml}^{-1}$ and then spin-coated at $2000 \mathrm{rpm}$ for $60 \mathrm{~s}$. After the dielectric-layer coating, the devices were baked at $80{ }^{\circ} \mathrm{C}$ for more than 2 hours in a nitrogen glove box. The OFETs were completed by the deposition of an Al gate electrode using a shadow mask.

\section{Characterizations of transistors}

The OFET electrical characteristics were measured using a Keithley 4200-SCS parameter analyzer in a nitrogen-filled glove box. The mobility and threshold voltage were calculated in the linear or saturation region using the gradual-channel approximation. $C-V$ characteristics were measured using an Agilent 4284 precision LCR meter and a Keithley 4200-SCS parameter analyzer. For the temperature measurements, the vacuum chamber sealed at $\sim 10^{-2}$ Torr was adjusted to $294-195 \mathrm{~K}$ using liquid nitrogen and $I-V$ results were obtained at each temperature.

\section{Acknowledgements}

The authors gratefully acknowledge the financial support of the project from the National Natural Science Foundation of China (61504173), Guangdong Provincial Department of Science and Technology (2015B090924001), the Guangdong Natural Science Funds for Distinguished Young Scholars under Grant 2016A030306046, and the Center for Advanced Soft-Electronics (2013M3A6A5073183) funded by the Ministry of Science, ICT \& Future Planning. CL \& YYN thank Dr Yong Xu for discussions.

\section{References}

1 (a) K. Nakayama, Y. Hirose, J. Soeda, M. Yoshizumi, T. Uemura, M. Uno, W. Li, M. J. Kang, M. Yamagishi, Y. Okada, E. Miyazaki, Y. Nakazawa, A. Nakao, K. Takimiya and J. Takeya, Adv. Mater., 2011, 23, 1626; (b) C. Liu, T. Minari, X. Lu, A. Kumatani, K. Takimiya and K. Tsukagoshi, Adv. Mater., 2011, 23, 523; (c) H. Dong, X. Fu, J. Liu, Z. Wang and W. Hu, Adv. Mater., 2013, 25, 6158.

2 (a) I. Kang, H.-J. Yun, D. S. Chung, S.-K. Kwon and Y.-H. Kim, J. Am. Chem. Soc., 2013, 135, 14896; (b) H.-R. Tseng, H. Phan, C. Luo, M. Wang, L. A. Perez, S. N. Patel, L. Ying, E. J. Kramer, T.-Q. Nguyen, G. C. Bazan and A. J. Heeger, Adv. Mater., 2014, 26, 2993; (c) G. Kim, S.-J. Kang, G. K. Dutta, Y.-K. Han, T. J. Shin, Y.-Y. Noh and C. Yang, J. Am. Chem. Soc., 2014, 136, 9477.

3 (a) Y. Li, P. Sonar, L. Murphy and W. Hong, Energy Environ. Sci., 2013, 6, 1684; (b) H. Bronstein, J. M. Frost, A. Hadipour, Y. Kim, C. B. Nielsen, R. S. Ashraf, B. P. Rand, S. Watkins and I. McCulloch, Chem. Mater., 2013, 25, 277; (c) J. D. Yuen and F. Wudl, Energy Environ. Sci., 2013, 6, 392. 
4 (a) A. Troisi, Chem. Soc. Rev., 2011, 40, 2347; (b) R. Noriega, J. Rivnay, K. Vandewal, F. P. V. Koch, N. Stingelin, P. Smith, M. F. Toney and A. Salleo, Nat. Mater., 2013, 12, 1038.

5 (a) G. Horowitz, J. Mater. Res., 2004, 19, 1946; (b) A. R. Murphy and J. M. J. Frechet, Chem. Inf., 2007, 38, 1066; (c) I. McCulloch, M. Heeney, C. Bailey, K. Genevicius, I. MacDonald, M. Shkunov, D. Sparrowe, S. Tierney, R. Wagner, W. Zhang, M. L. Chabinyc, R. J. Kline, M. D. McGehee and M. F. Toney, Nat. Mater., 2006, 5, 328.

6 (a) H. Sirringhaus, Adv. Mater., 2014, 26, 1319; (b) V. Coropceanu, J. Cornil, D. A. da Silva Filho, Y. Olivier, R. Silbey and J.-L. Brédas, Chem. Rev., 2007, 107, 926.

7 (a) I. N. Hulea, S. Fratini, H. Xie, C. L. Mulder, N. N. Iossad, G. Rastelli, S. Ciuchi and A. F. Morpurgo, Nat. Mater., 2006, 5, 982; (b) H. J. Ding, C. Reese, A. J. Makinen, Z. A. Bao and Y. L. Gao, Appl. Phys. Lett., 2010, 96, 222106; (c) S. Ciuchi, R. C. Hatch, H. Höchst, C. Faber, X. Blase and S. Fratini, Phys. Rev. Lett., 2012, 108, 256401.

8 D. Venkateshvaran, M. Nikolka, A. Sadhanala, V. Lemaur, M. Zelazny, M. Kepa, M. Hurhangee, A. J. Kronemeijer, V. Pecunia, I. Nasrallah, I. Romanov, K. Broch, I. McCulloch, D. Emin, Y. Olivier, J. Cornil, D. Beljonne and H. Sirringhaus, Nature, 2014, 515, 384.

9 (a) S. D. Brotherton, in Introduction to Thin Film Transistors: Physics and Technology of TFTs, Springer International Publishing, Heidelberg, 2013, p. 339; (b) J. Y. W. Seto, J. Appl. Phys., 1975, 46, 5247; (c) J. Levinson, F. R. Shepherd, P. J. Scanlon, W. D. Westwood, G. Este and M. Rider, J. Appl. Phys., 1982, 53, 1193; (d) A. T. Voutsas, IEEE Trans. Electron Devices, 2003, 50, 1494; (e) G. Horowitz, Adv. Mater., 1998, 10, 365; $(f)$ P. G. Le Comber and W. E. Spear, Phys. Rev. Lett., 1970, 25, 509.

10 (a) N. F. Mott, Philos. Mag., 1969, 19, 835; (b) M. C. J. M. Vissenberg and M. Matters, Phys. Rev. B: Condens. Matter Mater. Phys., 1998, 57, 12964; (c) S. D. Baranovskii, T. Faber, F. Hensel and P. Thomas, J. Phys.: Condens. Matter, 1997, 9, 2699; (d) N. F. Mott and E. A. Davis, Electronic processes in non-crystalline materials, Clarendon Press, 1979.

11 (a) H. Bässler, Phys. Status Solidi B, 1993, 175, 15; (b) R. H. Young, Philos. Mag. B, 1995, 72, 435; (c) R. Schmechel, Phys. Rev. B: Condens. Matter Mater. Phys., 2002, 66, 235206.

12 S. Lee, K. Ghaffarzadeh, A. Nathan, J. Robertson, S. Jeon, C. Kim, I.-H. Song and U.-I. Chung, Appl. Phys. Lett., 2011, 98, 203508.

13 (a) J. Yin, R.-F. Chen, S.-L. Zhang, H.-H. Li, G.-W. Zhang, X.-M. Feng, Q.-D. Ling and W. Huang, J. Phys. Chem. C, 2011, 115, 14778; (b) R. A. Marcus, J. Chem. Phys., 1956, 24, 966; (c) R. A. Marcus, Rev. Mod. Phys., 1993, 65, 599; (d) I. Yavuz, B. N. Martin, J. Park and K. N. Houk, J. Am. Chem. Soc., 2015, 137, 2856.

14 K. Asadi, A. J. Kronemeijer, T. Cramer, L. Jan Anton Koster, P. W. M. Blom and D. M. de Leeuw, Nat. Commun., 2013, 4, 1710 .

15 (a) N. J. van der Kaap, I. Katsouras, K. Asadi, P. W. M. Blom, L. J. A. Koster and D. M. de Leeuw, Phys. Rev. B: Condens. Matter Mater. Phys., 2016, 93, 140206; (b) G. Nan, X. Yang,
L. Wang, Z. Shuai and Y. Zhao, Phys. Rev. B: Condens. Matter Mater. Phys., 2009, 79, 115203; (c) Y. Jiang, Q. Peng, H. Geng, H. Ma and Z. Shuai, Phys. Chem. Chem. Phys., 2015, 17, 3273. 16 (a) Y. Jiang, X. Zhong, W. Shi, Q. Peng, H. Geng, Y. Zhao and Z. Shuai, Nanoscale Horiz., 2016, 1, 53; (b) H. Geng, Q. Peng, L. Wang, H. Li, Y. Liao, Z. Ma and Z. Shuai, Adv. Mater., 2012, 24, 3568.

17 Y. Roichman and N. Tessler, Appl. Phys. Lett., 2002, 80, 1948. 18 L. Li, N. Lu and M. Liu, J. Appl. Phys., 2014, 116, 164504.

19 (a) D. Khim, Y. Xu, K.-J. Baeg, M. Kang, W.-T. Park, S.-H. Lee, I.-B. Kim, J. Kim, D.-Y. Kim, C. Liu and Y.-Y. Noh, Adv. Mater., 2016, 28, 518; (b) Y. Xu, M. Benwadih, R. Gwoziecki, R. Coppard, T. Minari, C. Liu, K. Tsukagoshi, J. Chroboczek, F. Balestra and G. Ghibaudo, J. Appl. Phys., 2011, 110, 104513. 20 L. Wang, G. Nan, X. Yang, Q. Peng, Q. Li and Z. Shuai, Chem. Soc. Rev., 2010, 39, 423.

21 (a) T. Sakanoue and H. Sirringhaus, Nat. Mater., 2010, 9, 736; (b) V. Podzorov, E. Menard, A. Borissov, V. Kiryukhin, J. A. Rogers and M. E. Gershenson, Phys. Rev. Lett., 2004, 93, 086602; (c) N. A. Minder, S. Ono, Z. Chen, A. Facchetti and A. F. Morpurgo, Adv. Mater., 2012, 24, 503.

22 W. C. Germs, K. Guo, R. A. J. Janssen and M. Kemerink, Phys. Rev. Lett., 2012, 109, 016601.

23 A. J. Kronemeijer, V. Pecunia, D. Venkateshvaran, M. Nikolka, A. Sadhanala, J. Moriarty, M. Szumilo and H. Sirringhaus, Adv. Mater., 2014, 26, 728.

24 D. Knipp, R. A. Street and A. R. Völkel, Appl. Phys. Lett., 2003, 82, 3907.

25 H. Sirringhaus, T. Sakanoue and J.-F. Chang, Phys. Status Solidi B, 2012, 249, 1655.

26 R. J. Chesterfield, J. C. McKeen, C. R. Newman, P. C. Ewbank, D. A. da Silva Filho, J.-L. Brédas, L. L. Miller, K. R. Mann and C. D. Frisbie, J. Phys. Chem. B, 2004, 108, 19281.

27 J. Lee, A. R. Han, H. Yu, T. J. Shin, C. Yang and J. H. Oh, J. Am. Chem. Soc., 2013, 135, 9540.

28 Y. Yamashita, F. Hinkel, T. Marszalek, W. Zajaczkowski, W. Pisula, M. Baumgarten, H. Matsui, K. Müllen and J. Takeya, Chem. Mater., 2016, 28, 420.

29 J.-F. Chang, H. Sirringhaus, M. Giles, M. Heeney and I. McCulloch, Phys. Rev. B: Condens. Matter Mater. Phys., 2007, 76, 205204.

30 H. Sirringhaus, M. Bird and N. Zhao, Adv. Mater., 2010, 22, 3893.

31 M. Caironi, M. Bird, D. Fazzi, Z. Chen, R. Di Pietro, C. Newman, A. Facchetti and H. Sirringhaus, Adv. Funct. Mater., 2011, 21, 3371.

32 A. J. Kronemeijer, E. Gili, M. Shahid, J. Rivnay, A. Salleo, M. Heeney and H. Sirringhaus, Adv. Mater., 2012, 24, 1558.

33 S. Yadav, P. Kumar and S. Ghosh, Appl. Phys. Lett., 2012, 101, 193307.

34 T. D. Anthopoulos, D. M. d. Leeuw, E. Cantatore, P. v. t. Hof, J. Alma and J. C. Hummelen, J. Appl. Phys., 2005, 98, 054503.

35 S. F. Nelson, Y.-Y. Lin, D. J. Gundlach and T. N. Jackson, Appl. Phys. Lett., 1998, 72, 1854.

36 H. Sirringhaus, R. J. Wilson, R. H. Friend, M. Inbasekaran, W. Wu, E. P. Woo, M. Grell and D. D. C. Bradley, Appl. Phys. Lett., 2000, 77, 406. 
37 J. Veres, S. D. Ogier, S. W. Leeming, D. C. Cupertino and S. Mohialdin Khaffaf, Adv. Funct. Mater., 2003, 13, 199.

38 S. G. Bucella, A. Luzio, E. Gann, L. Thomsen, C. R. McNeill, G. Pace, A. Perinot, Z. Chen, A. Facchetti and M. Caironi, Nat. Commun., 2015, 6, 8394.
39 G. Horowitz, M. E. Hajlaoui and R. Hajlaoui, J. Appl. Phys., 2000, 87, 4456.

40 Y. Zhang, J. Qiao, S. Gao, F. Hu, D. He, B. Wu, Z. Yang, B. Xu, Y. Li, Y. Shi, W. Ji, P. Wang, X. Wang, M. Xiao, H. Xu, J.-B. Xu and X. Wang, Phys. Rev. Lett., 2016, 116, 016602. 